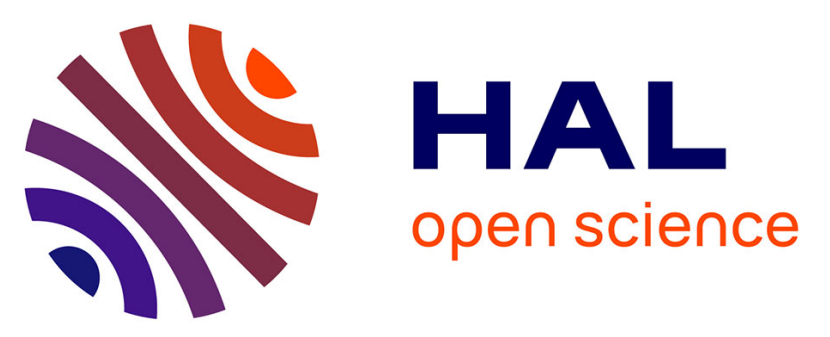

\title{
Response of a Periodically Supported Beam on a Non-Uniform Viscoelastic Foundation subject to Moving Loads
}

Tien Hoang, Denis Duhamel, Gilles Forêt, Honoré P. Yin, Gwendal Cumunel, P. Joyez, R. Caby

\section{To cite this version:}

Tien Hoang, Denis Duhamel, Gilles Forêt, Honoré P. Yin, Gwendal Cumunel, et al.. Response of a Periodically Supported Beam on a Non-Uniform Viscoelastic Foundation subject to Moving Loads. The Third International Conference on Railway Technology: Research, Development and Maintenance, Apr 2016, Cagliari, Italy. pp.185, 10.4203/ccp.110.185 . hal-01696135

\section{HAL Id: hal-01696135 https://hal.science/hal-01696135}

Submitted on 13 Feb 2018

HAL is a multi-disciplinary open access archive for the deposit and dissemination of scientific research documents, whether they are published or not. The documents may come from teaching and research institutions in France or abroad, or from public or private research centers.
L'archive ouverte pluridisciplinaire HAL, est destinée au dépôt et à la diffusion de documents scientifiques de niveau recherche, publiés ou non, émanant des établissements d'enseignement et de recherche français ou étrangers, des laboratoires publics ou privés. 


\title{
Response of a Periodically Supported Beam on a Non-uniform Viscoelastic Foundation Subjected to Moving Loads
}

\author{
T. Hoang ${ }^{1}$, D. Duhamel ${ }^{1}$, G. Foret ${ }^{1}$, H.P. Yin ${ }^{1}$, G. Cumunel ${ }^{1}$, P. Joyez ${ }^{2}$ \\ and R. Caby ${ }^{2}$ \\ ${ }^{1}$ University Paris-Est, Laboratory Navier, Ecole des Ponts ParisTech, \\ 6-8 avenue Blaise Pascal, Cité Descartes, Champs-sur-Marne, 77455 \\ Marne-la-Vallée Cedex 2, France
}

${ }^{2}$ Eurotunnel Group, BP no. 69, 62904 Coquelles Cedex, France

\begin{abstract}
The dynamics of railway tracks on non-uniform viscoelastic foundations is investigated by a new analytical model. Firstly, a model is developed for a periodically supported beam where the supports behaviours are changed periodically through the length of the beam. By using a periodicity condition, the dynamic equation of the Euler-Bernoulli beam leads to a general relation between the reaction forces of the supports and the displacement of the beam. Then, the responses are computed by combining this relation and the constitutive law of the supports. Thereafter, a periodically supported beam on a non-uniform foundation is considered with a support system included a defect zone where the supports behaviours are different from the non-defect supports. Then, a larger interval of the support system bounding this defect zone is considered as one period of a periodical support system provided that the dynamic responses outside this zone are unchanged and equal to the steady responses. The analytical method is efficient when the number of the supports in one period is not very large. Otherwise, a numerical method has been developed to compute the response by using the fast Fourier transforms and an iteration procedure. This method is simple and fast, particularly when using the fast Fourier transform, to compute the dynamic responses of railway tracks on non-uniform foundations.
\end{abstract}

Keywords: railway, non-uniform foundation, periodically supported beam, Dirac comb, fast Fourier transform.

\section{Introduction}

The dynamics of railway tracks have often been investigated by a periodically supported beam subjected to moving forces. In this model, the beam rests on identical supports at periodical intervals through the length of the beam. The supports can have 
linear or nonlinear behaviours which depend on the constitutive laws of the support components and the foundation. A spring-mass has been often used for such a support system in articles of Mead [1], Belotserkovskiy [2], Nordbord [3, 4]. In order to take into account the coupling of the supports via the foundation, Metrikine et al $[5,6]$ considered the shearing force of a viscoelastic foundation. Recently, Hoang et al. [7] has developed a periodicity condition to establish a general relation for any uniform support system. However, if the supports are not identical, these models are not applicable because the periodicity is not available. Unfortunately, the non-identical supports appear frequently, e.g. the transitions zones of a railway track, the damage of sleepers or foundation... and there are not any analytical model for this dynamical problem.

In this paper, we have developed an analytical model for a periodically supported beam subjected to moving forces with non-identical supports. In section 2, we present an analytical model for a periodical support system where the supports behaviours are changed periodically through the length of the beam. We propose a new periodicity condition for the reaction forces of the supports. This condition together with the Fourier transforms and the Dirac comb properties lead to a relation between the reaction forces of the supports and the displacement of the beam. Particularly, this relation can be described in a matrix form or in a convolution product form. Then, combining this relation with the constitutive laws of the support system gives the dynamical responses.

For a non-uniform support system, we consider a larger interval containing a defect zone. When this interval is large enough, we suppose that the dynamic responses are unchanged when the moving forces arrive or leave this interval, and they equal to the response of a support in an uniform support system. Therefore, we can consider this interval as one period of a periodical support system except that it contains many supports. Therefore, the previous model can be used. A numerical method is developed to calculated fast the responses based on the fast Fourier transforms and an iteration procedure. The numerical applications will be presented in section 3 with results of the analytical and numerical methods. The results shows that a support can be overloaded when the neighbour supports are damaged. This overloading is also studied for a railways track which misses some sleepers.

\section{Model}

\subsection{Dynamic stiffness of a support}

A support on a viscoelastic foundation can be described by a mass-spring system and the dynamic stiffness is given by

$$
K_{f}=k_{f}+\mathrm{i} \omega \eta_{f}-M \omega^{2}
$$

where $M$ is the mass of the support and $k_{f}, \eta_{f}$ are stiffness and damping coefficient of the foundation.In addition, the dynamic stiffness of the rail pad is $K_{p}=k_{p}+\mathrm{i} \omega \eta_{p}$. 
When the rail pad is included in a support system, the total dynamics stiffness of a support is calculated by

$$
\frac{1}{K_{s}}=\frac{1}{K_{f}}+\frac{1}{K_{p}}
$$

In other words, we can write

$$
K_{s}=\frac{\left(k_{f}+\mathrm{i} \omega \eta_{f}-M \omega^{2}\right)\left(k_{p}+\mathrm{i} \omega \eta_{p}\right)}{k_{f}+k_{p}+\mathrm{i} \omega\left(\eta_{p}+\eta_{p}\right)-M \omega^{2}}
$$

When an infinite beam rest on identical support at periodical intervals (called an uniform support system), we have a classical model for a periodically supported beam. This model has an exact analytical solution when it is subjected by moving forces. However, when the supports are not identical, i.e. $K_{s}$ are different from each others, this model is not applicable and there is no model for this dynamic problem. In addition, the difference of supports behaviours is very common phenomenon, e.g, the supports on a railway bridge, the transition zone between ballast and non-ballast railway tracks, the defect of the foundation and the sleepers... In the next sections, we will consider a support system where the supports behaviours are changed periodically through the length of the beam (in the section 2.2) and then a support system which contains a defect zone (in the section 2.3).

\subsection{Periodical support system}

Let's consider a beam rested on a periodical support system as shown in Figure 1. This system contains $m$ different supports separated by distance $l$ and they are distributed periodically through the length of the beam. Thus, this support system together with the beam is a periodic structure with the length of one period equals to $L=m l$. The beam is subjected by moving forces $Q_{j}$ which are characterized by the distances to the first moving force $D_{j}$.

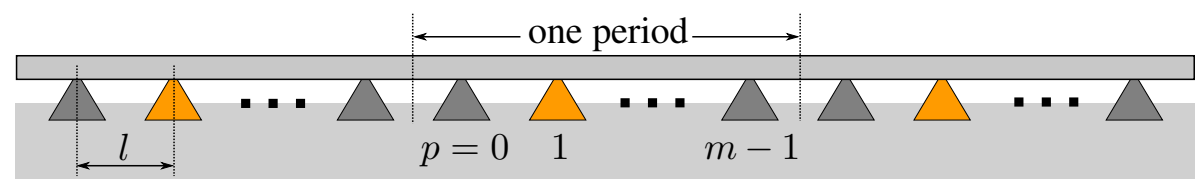

Figure 1: Beam on a periodical support system

In steady-state, we suppose that the responses of the support system are repeated when the forces move though a length equal to one period of the support system. It means that the reaction forces of two supports at the distance $L=m l$ are described by the same function with a delay equal to the time for the movement of the forces from one to another. In other words, the reaction force of the support $p+n m$ (with $n \in \mathbb{Z})$ at the coordinate $x=(p+n m) l$ is calculated by

$$
R_{n m+p}=R_{p}\left(t-\frac{n L}{v}\right) \quad \forall n \in \mathbb{Z}
$$


where $R_{p}(t)$ (with $0 \leq p<m$ ) is the reaction force of the support $p$ at the coordinate $x=p l$. By the help of the Dirac functions, the total of the reaction forces and the moving forces can be written as follows

$$
F(x, t)=\sum_{n \in \mathbb{Z}} \sum_{p=0}^{m-1} R_{p}\left(t-\frac{x-p l}{v}\right) \delta(x-p l-n L)-\sum_{j=1}^{K} Q_{j} \delta\left(x-D_{j}-v t\right)
$$

The dynamic equation of the Euler-Bernoulli beam subjected to force $F(x, t)$ is

$$
E I \frac{\partial^{4} w_{r}(x, t)}{\partial x^{4}}+\rho S \frac{\partial^{2} w_{r}(x, t)}{\partial t^{2}}=F(x, t)
$$

where $\rho, E$ are the density, the Young's modulus and $S, I$ are the section and the longitudinal inertia of the beam. By performing the Fourier transform of equations (5) and (6) with regard to time $t$, we obtain

$$
\begin{aligned}
& \hat{F}(x, \omega)=\sum_{n \in \mathbb{Z}} \sum_{p=0}^{m-1} \hat{R}_{p}(\omega) \mathrm{e}^{-\mathrm{i} \omega \frac{x-p l}{v}} \delta(x-n L-p l)-\sum_{j=1}^{K} \frac{Q_{j}}{v} \mathrm{e}^{-\mathrm{i} \omega \frac{x+D_{j}}{v}} \\
& E I \frac{\partial^{4} \hat{w}_{r}(x, \omega)}{\partial x^{4}}-\rho S \omega^{2} \hat{w}_{r}(x, \omega)=\hat{F}(x, \omega)+\hat{Q}(x, \omega)
\end{aligned}
$$

Thereafter, by combining the last equations and by performing the Fourier transform with regard to $x$, we obtain

$$
\begin{aligned}
\left(E I \lambda^{4}-\rho S \omega^{2}\right) \Pi(\lambda, \omega) & +2 \pi \delta\left(\lambda+\frac{\omega}{v}\right) \sum_{j=1}^{K} \frac{Q_{j}}{v} \mathrm{e}^{-\mathrm{i} \frac{\omega}{v} D_{j}} \\
- & \sum_{n \in \mathbb{Z}} \sum_{p=0}^{m-1} \hat{R}_{p}(\omega) \mathrm{e}^{-\mathrm{i}\left(\lambda+\frac{\omega}{v}\right) n L-\mathrm{i} \lambda p l}=0
\end{aligned}
$$

where $\Pi(\lambda, \omega)$ is the Fourier transform of $\hat{w}_{r}(x, \omega)$ with regard to $x$. The last term of equation (7) can be rewritten as follows

$$
\sum_{n \in \mathbb{Z}} \sum_{p=0}^{m-1} \hat{R}_{p}(\omega) \mathrm{e}^{-\mathrm{i}\left(\lambda+\frac{\omega}{v}\right) n L-i \lambda p l}=\left(\sum_{p=0}^{m-1} \hat{R}_{p}(\omega) \mathrm{e}^{-\mathrm{i} \lambda p l}\right) \sum_{n \in \mathbb{Z}} \mathrm{e}^{-\mathrm{i}\left(\lambda+\frac{\omega}{v}\right) n L}
$$

By using the properties of the Dirac comb [8], we have

$$
\sum_{n \in \mathbb{Z}} \mathrm{e}^{-\mathrm{i}\left(\lambda+\frac{\omega}{v}\right) n L}=\frac{2 \pi}{L} \sum_{n \in \mathbb{Z}} \delta\left(\lambda+\frac{\omega}{v}+\frac{2 \pi n}{L}\right)
$$

By substituting equations (8), (9) into equation (7), we can write

$$
\Pi(\lambda, \omega)=\frac{2 \pi \hat{R}(\lambda, \omega)}{L E I\left(\lambda^{4}-\lambda_{e}^{4}\right)} \sum_{n \in \mathbb{Z}} \delta\left(\lambda+\frac{\omega}{v}+\frac{2 \pi n}{L}\right)-\frac{2 \pi \delta\left(\lambda+\frac{\omega}{v}\right)}{v E I\left(\lambda^{4}-\lambda_{e}^{4}\right)} \sum_{j=1}^{K} Q_{j} \mathrm{e}^{-\mathrm{i} \frac{\omega}{v} D_{j}}
$$


where $\lambda_{e}=\sqrt[4]{\frac{\rho S \omega^{2}}{E I}}$ and $\hat{R}(\lambda, \omega)$ is defined by

$$
\hat{R}(\lambda, \omega)=\sum_{p=0}^{m-1} \hat{R}_{p}(\omega) \mathrm{e}^{-\mathrm{i} \lambda p l}
$$

Finally, performing the inverse Fourier transform of $\Pi(\lambda, \omega)$ in equation (10) gives the following result

$$
\hat{w}_{r}(x, \omega)=\sum_{n \in \mathbb{Z}} \sum_{p=0}^{m-1} \frac{\hat{R}_{p}(\omega)}{L E I} \frac{\mathrm{e}^{-\mathrm{i}\left(\frac{2 \pi n}{L}+\frac{\omega}{v}\right)(x-p l)}}{\left(\frac{\omega}{v}+\frac{2 \pi n}{L}\right)^{4}-\lambda_{e}^{4}}-\sum_{j=1}^{K} \frac{Q_{j}}{v E I} \frac{\mathrm{e}^{-\mathrm{i} \omega \frac{x+D_{j}}{v}}}{\left(\frac{\omega}{v}\right)^{4}-\lambda_{e}^{4}}
$$

Equation (12) establishes a relation between the beam displacement and the reaction forces of the supports in steady-state. We will simplify this relation by computing the displacements of the beam at the support positions. For the support at the origin of reference, by substituting $x=0$ into the last equation, we obtain

$$
\begin{aligned}
\hat{w}_{r}(0, \omega) & =\sum_{n \in \mathbb{Z}} \sum_{p=0}^{m-1} \frac{\hat{R}_{p}(\omega)}{L E I} \frac{\mathrm{e}^{\mathrm{i}\left(\frac{2 \pi n}{L}+\frac{\omega}{v}\right) p l}}{\left(\frac{\omega}{v}+\frac{2 \pi n}{L}\right)^{4}-\lambda_{e}^{4}}-\sum_{j=1}^{K} \frac{Q_{j} \mathrm{e}^{-\mathrm{i} \frac{\omega}{v} D_{j}}}{v E I\left[\left(\frac{\omega}{v}\right)^{4}-\lambda_{e}^{4}\right]} \\
& =\sum_{p=0}^{m-1} \hat{R}_{p}(\omega) \mathrm{e}^{\mathrm{i} \frac{\omega}{v} p l} \eta_{p}-\eta_{0} \mathbf{Q}_{e}
\end{aligned}
$$

where

$$
\begin{aligned}
\eta_{p} & =\frac{1}{L E I} \sum_{n \in \mathbb{Z}} \frac{\mathrm{e}^{\mathrm{i} 2 \pi n \frac{p}{m}}}{\left(\frac{\omega}{v}+\frac{2 \pi n}{L}\right)^{4}-\lambda_{e}^{4}} \\
\mathrm{Q}_{e} & =\frac{1}{\eta_{0} v E I} \sum_{j=1}^{K} \frac{Q_{j} \mathrm{e}^{-\mathrm{i} \frac{\omega}{v} D_{j}}}{\left(\frac{\omega}{v}\right)^{4}-\lambda_{e}^{4}}
\end{aligned}
$$

This $\eta_{p}$ can be deduced to a simple analytical expression as shown in Appendix A.

For the support at $x=q l(0<q<m)$, we have

$$
\hat{w}_{r}(q l, \omega)=\sum_{n \in \mathbb{Z}} \sum_{p=0}^{m-1} \frac{\hat{R}_{p}(\omega)}{L E I} \frac{\mathrm{e}^{-\mathrm{i}\left(\frac{2 \pi n}{L}+\frac{\omega}{v}\right)(q-p) l}}{\left(\frac{\omega}{v}+\frac{2 \pi n}{L}\right)^{4}-\lambda_{e}^{4}}-\sum_{j=1}^{K} \frac{Q_{j}}{v E I} \frac{\mathrm{e}^{-\mathrm{i} \frac{\omega}{v}\left(q l+D_{j}\right)}}{\left(\frac{\omega}{v}\right)^{4}-\lambda_{e}^{4}}
$$

By multiplying two sides of the last equation with $\mathrm{e}^{\mathrm{i} \omega \frac{q l}{v}}$, we obtain

$$
\hat{w}_{r}(q l, \omega) \mathrm{e}^{\mathrm{i} \omega \frac{q l}{v}}=\sum_{p=0}^{m-1} \sum_{n \in \mathbb{Z}} \frac{\hat{R}_{p}(\omega) \mathrm{e}^{\mathrm{i} \omega \frac{p l}{v}}}{L E I} \frac{\mathrm{e}^{-\mathrm{i} 2 \pi n \frac{q-p}{m}}}{\left(\frac{\omega}{v}+\frac{2 \pi n}{L}\right)^{4}-\lambda_{e}^{4}}-\sum_{j=1}^{K} \frac{Q_{j}}{v E I} \frac{\mathrm{e}^{-\mathrm{i} \frac{\omega}{v} D_{j}}}{\left(\frac{\omega}{v}\right)^{4}-\lambda_{e}^{4}}
$$

By using the notation $\eta_{(p-q)}$ defined by equation (14) with a remark that $\eta_{(p-q)}=$ $\eta_{(m+p-q)}$, the last equation becomes

$$
\hat{w}_{r}(q l, \omega) \mathrm{e}^{\mathrm{i} \omega \frac{q l}{v}}=\sum_{p=0}^{m-1} \hat{R}_{p}(\omega) \mathrm{e}^{\mathrm{i} \omega \frac{p l}{v}} \eta_{(p-q)}-\eta_{0} \mathbf{Q}_{e}
$$


Let $\mathbf{w}_{q}(t)$ and $\mathbf{R}_{q}(t)$ be defined by

$$
\left\{\begin{array}{l}
\mathbf{w}_{q}(t)=w_{r}\left(q l, t-\frac{q l}{v}\right) \\
\mathbf{R}_{q}(t)=R_{q}\left(t-\frac{q l}{v}\right)
\end{array}\right.
$$

Indeed, $\mathbf{w}_{q}$ and $\mathbf{R}_{q}$ are the displacements and the reaction force at $x=q l$ with the time $t=0$ corresponds to the instance that the first moving force arrives this position. By performing the Fourier transform of the last equations, we obtain

$$
\left\{\begin{array}{l}
\hat{\mathbf{w}}_{q}(\omega)=\hat{w}_{r}(q l, \omega) \mathrm{e}^{\mathrm{i} \omega \frac{q l}{v}} \\
\hat{\mathbf{R}}_{q}(\omega)=\hat{R}_{q}(\omega) \mathrm{e}^{\mathrm{i} \omega \frac{q l}{v}}
\end{array}\right.
$$

By substituting equation (18) into equation (16), we have

$$
\hat{\mathbf{w}}_{q}=\sum_{p=0}^{m-1} \hat{\mathbf{R}}_{p} \eta_{(p-q)}-\eta_{0} \mathbf{Q}_{e}
$$

The last equation can be written in matrix form as follows

$$
\left(\begin{array}{cccc}
\eta_{0} & \eta_{1} & \cdots & \eta_{m-1} \\
\eta_{m-1} & \eta_{0} & \cdots & \eta_{m-2} \\
\vdots & \vdots & \ddots & \vdots \\
\eta_{1} & \eta_{2} & \cdots & \eta_{0}
\end{array}\right)\left(\begin{array}{c}
\hat{\mathbf{R}}_{0} \\
\hat{\mathbf{R}}_{1} \\
\vdots \\
\hat{\mathbf{R}}_{m-1}
\end{array}\right)=\eta_{0} \mathbf{Q}_{e}\left(\begin{array}{c}
1 \\
1 \\
\vdots \\
1
\end{array}\right)+\left(\begin{array}{c}
\hat{\mathbf{w}}_{e} \\
\hat{\mathbf{w}}_{e} \\
\vdots \\
\hat{\mathbf{w}}_{e}
\end{array}\right)
$$

or

$$
\underline{\underline{\mathbf{C}}} \underline{\hat{\mathbf{R}}}=\eta_{0} \mathbf{Q}_{e} \underline{\mathbf{I}}+\underline{\hat{\mathbf{w}}}
$$

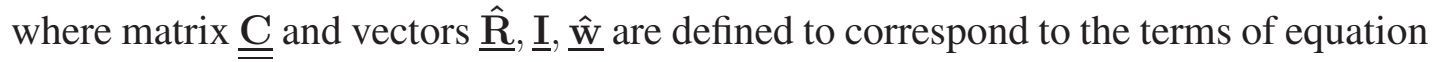
(20).

Equation (21) is another relation between the reaction forces of the supports and the displacements of the beam at the support positions; This relation does not depend on the supports behaviours, and it is the result of the periodicity condition and the dynamic equation of the Euler-Bernoulli beam. We will now combine this relation with the constitutive laws of the support system to work out the responds.

Suppose that all supports are linear supports as shown in section 2.1. Let $k_{0}, k_{1}, \ldots, k_{m-1}$ be dynamic stiffness of $m$ supports in one period of the support system. By definition, we have

$$
\hat{\mathbf{R}}_{q}(\omega)=-k_{q} \hat{\mathbf{w}}_{q}(\omega)
$$

The last equation can be written in the matrix form following:

$$
\underline{\hat{\mathbf{w}}}=-\underline{\underline{\mathbf{D}}} \underline{\hat{\mathbf{R}}}
$$

where $\underline{\underline{\mathrm{D}}}$ is defined by

$$
\underline{\underline{\mathbf{D}}}=\left(\begin{array}{cccc}
1 / k_{0} & 0 & \cdots & 0 \\
0 & 1 / k_{1} & \cdots & 0 \\
\vdots & \vdots & \ddots & \vdots \\
0 & 0 & \cdots & 1 / k_{m-1}
\end{array}\right)=\operatorname{diag}\left(1 / k_{0}, 1 / k_{1}, \ldots 1 / k_{m-1}\right)
$$


Then, by combining equations (21) and (23), we obtain

$$
\eta_{0}^{-1}(\underline{\underline{\mathbf{C}}}+\underline{\underline{\mathbf{D}}}) \underline{\hat{\mathbf{R}}}=\mathbf{Q}_{e} \underline{\mathbf{I}}
$$

The matrix $\underline{\underline{\mathbf{A}}}=\eta_{0}^{-1}(\underline{\underline{\mathbf{C}}}+\underline{\underline{\mathbf{D}}})$ can be written as follows

$$
\underline{\underline{\mathbf{A}}}=\left(\begin{array}{cccc}
1+1 / \eta_{0} k_{0} & \eta_{1} / \eta_{0} & \cdots & \eta_{m-1} / \eta_{0} \\
\eta_{m-1} / \eta_{0} & 1+1 / \eta_{0} k_{1} & \cdots & \eta_{m-2} / \eta_{0} \\
\vdots & \vdots & \ddots & \vdots \\
\eta_{1} / \eta_{0} & \eta_{2} / \eta_{0} & \cdots & 1+1 / \eta_{0} k_{m-1}
\end{array}\right)
$$

Finally, the reaction forces are obtained by

$$
\underline{\hat{\mathbf{R}}}=\mathrm{Q}_{e} \underline{\underline{\mathbf{A}}}^{-1} \underline{\mathbf{I}}
$$

Wee see that $\underline{\underline{\mathbf{A}}}$ is dimensionless $m \times m$ matrix and it depends on the frequency $\omega$. Therefore, we have to inverse this matrix for all frequencies to find out the reaction forces of the supports given by equation (27). This calculation is simple and fast when $m$ is small. However, if it exists a stiffness equals to zero (e.g. lack of a sleeper in a railway track), $\underline{\underline{\mathbf{A}}}$ is not convergent and equation (27) can not be applied. In the next section, we will present a numerical method to compute the responses. This method is convenient for computing a big number of supports in one period which is presented a support system containing a defect zone.

\subsection{Non-uniform support system}

Let's consider a periodically supported beam which has a defect zone where the supports behaviours are different from others zones. This zone is located in a interval contained $m$ supports (see Figure 2). $m$ is supposed big enough for that the responses are unchanged when the moving forces come or leave this interval (and they equal to the responses of an uniform supports). Thus, we can consider this interval as one period of a periodic support system as shown in the previous section. Therefore, we can use equation (27) to compute the response of the supports system. In this section, we will develop a numerical method for equations (19) and (22) to compute the responses of the supports system.

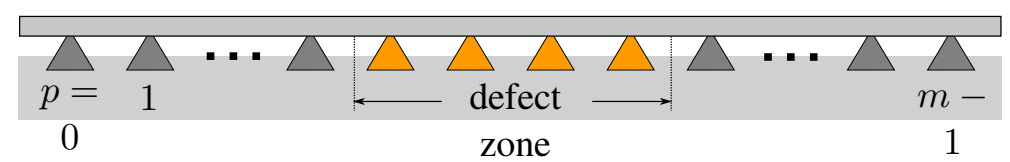

Figure 2: Periodically supported beam included a defect zone

Equation (19) presents a convolution product and it can be written by

$$
\underline{\hat{\mathbf{w}}}=\underline{\eta} \star \underline{\hat{\mathbf{R}}}-\eta_{0} \mathbf{Q}_{e} \underline{\mathbf{I}}
$$


where $\underline{\eta}=\left(\eta_{0} \eta_{1} \cdots \eta_{m-1}\right)^{t}$. Performing the discrete Fourier transform of the last equation gives

$$
\mathcal{F}_{q}\{\underline{\hat{\mathbf{w}}}\}=\mathcal{F}_{q}\{\underline{\eta}\} \mathcal{F}_{q}\{\underline{\hat{\mathbf{R}}}\}-\eta_{0} \mathbf{Q}_{e} \mathcal{F}_{q}\{\underline{\mathbf{I}}\}
$$

where the discrete Fourier transform of a vector (e.g. $\underline{\hat{\mathbf{w}}})$ is defined by

$$
\mathcal{F}_{q}\{\underline{\hat{\mathbf{w}}}\}=\sum_{p=0}^{m-1} \mathrm{e}^{i 2 \pi \frac{p q}{m}} \hat{\mathbf{w}}_{p}
$$

In addition, we have (see Appendix B)

$$
\mathcal{F}_{q}\{\underline{\eta}\}=\chi_{q}=\frac{1}{4 \lambda_{e}^{3} E I}\left(\frac{\sin l \lambda_{e}}{\cos l \lambda_{e}-\cos \left(\frac{\omega l}{v}+\frac{2 \pi q}{m}\right)}-\frac{\sinh l \lambda_{e}}{\cosh l \lambda_{e}-\cos \left(\frac{\omega l}{v}+\frac{2 \pi q}{m}\right)}\right)
$$

Thus, equation (29) becomes

$$
\mathcal{F}_{q}\{\underline{\hat{\mathbf{w}}}\}=\chi_{q} \mathcal{F}_{q}\{\underline{\hat{\mathbf{R}}}\}-m \eta_{0} \mathbf{Q}_{e} \delta_{1 q}
$$

or we can write:

$$
\mathcal{F}_{q}\{\underline{\hat{\mathbf{R}}}\}=\kappa_{q} \mathcal{F}_{q}\{\underline{\hat{\mathbf{w}}}\}+m \mathcal{Q}_{e} \delta_{1 q}
$$

where $\kappa_{q}=\chi_{q}^{-1}$ and $\mathcal{Q}_{e}=\eta_{0} \mathbf{Q}_{e} / \chi_{0}$.

Equation (31) is a characteristic relation of a periodically supported beam subjected to moving forces. This equation links the discrete Fourier transforms of the series of the reaction forces and the series of the beam displacements at support positions. When all supports are identical, the vectors of reactions forces and displacements are proportional to $\underline{I}$ and we can deduce easily this quation to

$$
\hat{\mathbf{R}}_{0}=\kappa_{0} \hat{\mathbf{w}}_{0}-\mathcal{Q}_{e}
$$

The last equation is a fundamental relation of a periodically supported beam with identical supports presented by Hoang et al. [7].

We will now combine this equation with the constitutive laws of the supports to find out the responses. We consider the system of linear supports described by equation (22). This equation can be also written as follows

$$
\underline{\hat{\mathbf{R}}}=-\underline{\underline{\mathbf{K}}} \underline{\hat{\mathbf{w}}}
$$

where $\underline{\underline{\mathbf{K}}}=\operatorname{diag}\left(k_{0}, k_{1}, \ldots, k_{m-1}\right)$. The last equation can be rewritten by

$$
\begin{aligned}
\underline{\hat{\mathbf{R}}} & =-\left(\underline{\underline{\mathbf{K}}}-k_{n d} \underline{\mathbf{I}}\right) \underline{\hat{\mathbf{w}}}-k_{n d} \underline{\hat{\mathbf{w}}} \\
& =\underline{\mathbf{R}}-k_{n d} \underline{\hat{\mathbf{w}}}
\end{aligned}
$$

where $k_{n d}$ is the dynamic stiffness of a non-defect support.By substituting equation (35) into equation (31), we obtain

$$
\left\{\begin{array}{l}
\underline{\tilde{\mathbf{R}}}=-\left(\underline{\left.\underline{\mathbf{K}}-k_{n d} \underline{\mathbf{I}}\right)} \underline{\hat{\mathbf{w}}}\right. \\
\mathcal{F}_{q}\{\underline{\hat{\mathbf{w}}}\}=\frac{1}{\kappa_{q}+k_{n d}}\left[\mathcal{F}_{q}\{\underline{\tilde{\mathbf{R}}}\}+m \mathcal{Q}_{e} \delta_{1 q}\right]
\end{array}\right.
$$




\begin{tabular}{|l|c|c|c|}
\hline Rail mass & $\rho S$ & $\mathrm{kgm}^{-1}$ & 60 \\
Rail stiffness & $E I$ & $\mathrm{MNm}^{2}$ & 6.3 \\
Train speed & $v$ & $\mathrm{~km} / \mathrm{h}$ & 160 \\
Charge per wheel & $Q$ & $\mathrm{kN}$ & 100 \\
Block mass & $M$ & $\mathrm{~kg}$ & 90 \\
Sleeper length & $l$ & $\mathrm{~m}$ & 0.6 \\
Stiffness of rail pad & $k_{p}$ & $\mathrm{MNm}^{-1}$ & 200 \\
Damping coefficient of rail pad & $\eta_{p}$ & $\mathrm{MNsm}^{-1}$ & 1.0 \\
Stiffness of prepared sleeper & $k_{f}$ & $\mathrm{MNm}^{-1}$ & 20 \\
Damping coefficient of prepared sleeper & $\eta_{f}$ & $\mathrm{MNsm}^{-1}$ & 0.2 \\
Stiffness of damaged sleeper & $k_{f}^{\prime}$ & $\mathrm{MNm}^{-1}$ & 10 \\
Damping coefficient of damaged sleeper & $\eta_{f}^{\prime}$ & $\mathrm{MNsm}^{-1}$ & 0.2 \\
\hline
\end{tabular}

Table 1: Parameters of a railway track

Remarks: $k_{q}=k_{n d}$ when the support $q$ is outside of the defect zone. Thus, the vector $\underline{\tilde{R}}$ takes zeros for all components outside of the defect zone.

Iteration procedure: Let's add an evolution index into equation (36) as follows

$$
\left\{\begin{array}{l}
\underline{\tilde{\mathbf{R}}}^{n}=-\left(\underline{\underline{\mathbf{K}}}-k_{n d} \underline{\underline{\mathbf{I}}}\right) \underline{\hat{\mathbf{w}}}^{n} \\
\underline{\hat{\mathbf{w}}}^{n+1}=\mathcal{F}^{-1}\left(\frac{1}{\kappa_{q}+k_{n d}}\left[\mathcal{F}_{q}\left(\underline{\tilde{\mathbf{R}}}^{n}\right)+m \mathcal{Q}_{e} \delta_{1 q}\right]_{0 \leq q<m}\right)
\end{array}\right.
$$

where the initial values are given as the response of a periodically supported beam with an uniform support system

$$
\underline{\hat{\mathbf{w}}}^{0}=\frac{\mathcal{Q}_{e}}{\kappa_{0}+k_{s}} \underline{\mathbf{I}}
$$

Equation (37) and (38) define completely a recurrent sequences of an iteration procedure. This procedure gives an approximation of the displacement vector $\underline{\hat{\mathbf{w}}}$. The reaction forces is computed from this vector by equation (33).

\section{Numerical examples}

\subsection{Prepared railway track with periodical replacement}

In order to prepare fast the damaged railway sleepers without many traffic interruptions, people can chose to replace a damaged sleeper among 3 successive damaged sleepers. Therefore, this procedure makes a periodic support system as mentioned in section 2.2 where one period contains one prepared support and two damaged supports. Let's consider such a prepared railway track with parameters given in Table 1. 
By using equation (3), the dynamic stiffness of supports in one period are

$$
\begin{array}{r}
k_{1}=K_{\text {prepared }}=\frac{\left(k_{f}+\mathrm{i} \omega \eta_{f}-M \omega^{2}\right)\left(k_{p}+\mathrm{i} \omega \eta_{p}\right)}{k_{f}+k_{p}+\mathrm{i} \omega\left(\eta_{f}+\eta_{p}\right)-M \omega^{2}} \\
k_{2}=k_{3}=K_{\text {damaged }}=\frac{\left(k_{f}^{\prime}+\mathrm{i} \omega \eta_{f}^{\prime}-M \omega^{2}\right)\left(k_{p}+\mathrm{i} \omega \eta_{p}\right)}{k_{f}^{\prime}+k_{p}+\mathrm{i} \omega\left(\eta_{f}^{\prime}+\eta_{p}\right)-M \omega^{2}}
\end{array}
$$

We calculate the responses of the sleepers by using the analytic formula (27). Figures 3 and 4 show the responses of the sleepers in one period of railway track. In these figures, the "reference" curves correspond to the responses of a railway track with all sleepers are prepared $\left(k_{1}=k_{2}=k_{3}=K_{\text {prepared }}\right)$. Here we have changed the time scale so that the time $t=0$ corresponds to the moment where the moving force is at the sleeper position. We see that the displacements of all sleepers are almost the

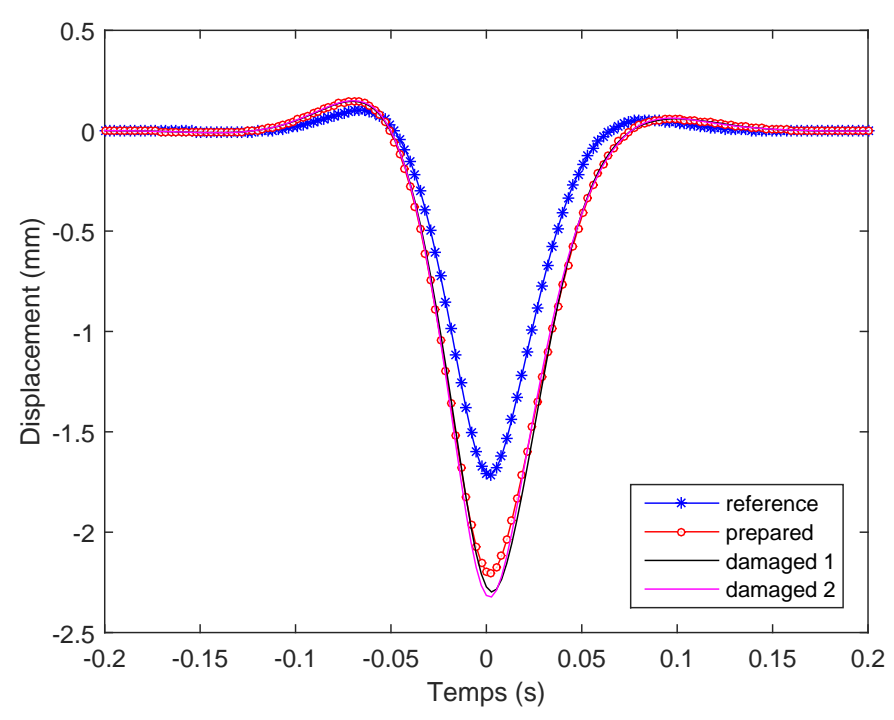

Figure 3: Displacements of the sleepers

same but they are bigger than the reference displacement. Indeed, the stiffness of the damaged sleepers are smaller than the reference stiffness and it causes this difference. Otherwise, the reaction forces of the supports are very different from each other. The prepared sleeper is overloading while the damaged sleepers are under-loading. In conclusion, this periodical replacement can improve the stiffness of the railway track but it makes the prepared supports are overloading and they could be damaged soon.

\subsection{Railway track with defect zone}

When a railway track misses some sleepers, the absent sleepers can be consider as a defect zone with the dynamic stiffness equal to zeros $k_{\text {damaged }}=0$. In this example, we consider the railway track given in Table 1 with one defect sleeper. We take the numerical method with an interval $m=101$ supports which contains the defect sleeper 


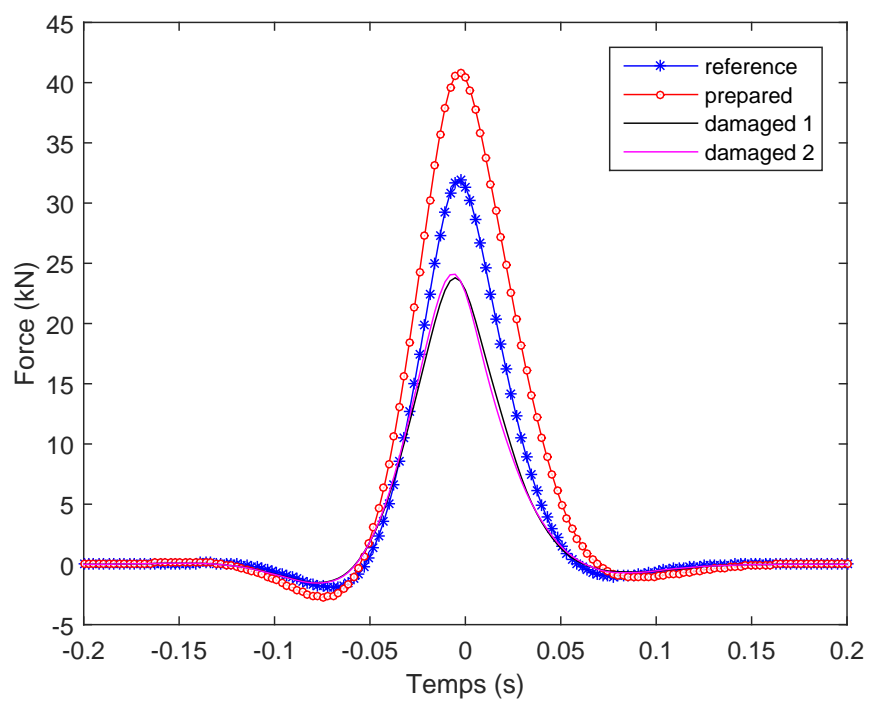

Figure 4: Reaction forces of the sleepers

at the middle. Figures 5 and 6 show the responds of the supports in this interval. We see that the dynamical influence of the defect sleeper is negligible from the third next supports of the defect sleeper. This result verifies again the condition that the responds are unchanged outside the interval and they equal to the reference response. Moreover, the first and the second next sleepers to the defect sleeper are overloading and the displacement of the beam at the defect sleeper increases significantly.

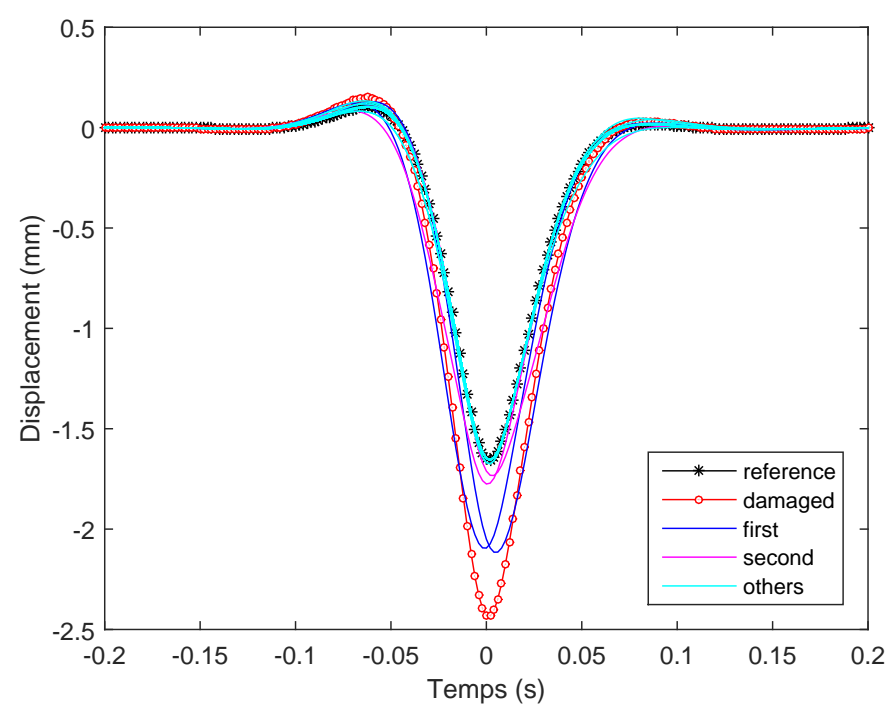

Figure 5: Displacements of the sleepers 


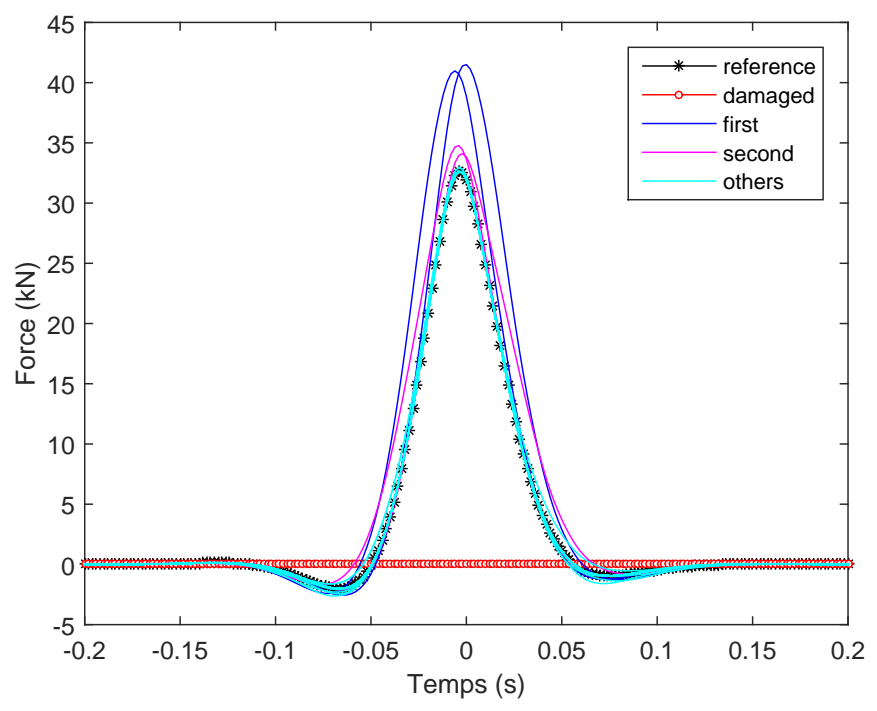

Figure 6: Reaction forces of the sleepers

\section{Conclusion}

An analytical model for a railway track with a non-uniform support system has been developed. By using a periodicity condition, the dynamic equation of the EulerBernoulli beam leads to a relation between the beam displacement and the reaction forces of the supports. Then, the analytical and numerical methods have been developed to find out the dynamical responses. This model has been applied to the dynamics of railway tracks with different kinds of support system. The calculations are very fast, especially when using the numerical method.

\section{Appexndix A. Calculation of expression $\eta_{p}$}

We will reduce a more general expression of $\eta_{p}$ defined as follows

$$
\begin{aligned}
\eta(x) & =\frac{1}{L E I} \sum_{n \in \mathbb{Z}} \frac{\mathrm{e}^{-\mathrm{i} 2 \pi n \frac{x}{L}}}{\left(\frac{\omega}{v}+\frac{2 \pi n}{L}\right)^{4}-\lambda_{e}^{4}} \\
& =\frac{L}{2 \lambda_{e}^{2} E I} \sum_{n \in \mathbb{Z}}\left[\frac{\mathrm{e}^{-\mathrm{i} 2 \pi n \frac{x}{L}}}{\left(\frac{\omega L}{v}+2 \pi n\right)^{2}-\left(L \lambda_{e}\right)^{2}}-\frac{\mathrm{e}^{-\mathrm{i} 2 \pi n \frac{x}{L}}}{\left(\frac{\omega L}{v}+2 \pi n\right)^{2}+\left(L \lambda_{e}\right)^{2}}\right]
\end{aligned}
$$

We will show that each term of the last expression can be deduced as follows:

$$
\begin{gathered}
\sum_{n \in \mathbb{Z}} \frac{\mathrm{e}^{-\mathrm{i} 2 \pi n \frac{x}{L}}}{\left(\frac{\omega L}{v}+2 \pi n\right)^{2}-\left(L \lambda_{e}\right)^{2}}=\frac{\mathrm{e}^{\mathrm{i} \omega \frac{x}{v}} \sin \lambda_{e}(L-x)+\mathrm{e}^{-\mathrm{i} \omega \frac{L-x}{v}} \sin \lambda_{e} x}{2 L \lambda_{e}\left(\cos L \lambda_{e}-\cos \frac{\omega L}{v}\right)} \\
\sum_{n \in \mathbb{Z}} \frac{\mathrm{e}^{-\mathrm{i} 2 \pi n \frac{x}{L}}}{\left(\frac{\omega L}{v}+2 \pi n\right)^{2}+\left(L \lambda_{e}\right)^{2}}=\frac{\mathrm{e}^{\mathrm{i} \omega \frac{x}{v}} \sinh \lambda_{e}(L-x)+\mathrm{e}^{-\mathrm{i} \omega \frac{L-x}{v}} \sinh \lambda_{e} x}{2 L \lambda_{e}\left(\cosh L \lambda_{e}-\cos \frac{\omega L}{v}\right)}
\end{gathered}
$$


Indeed, the right hand sides of equations (42) and (43) are expressions of Fourier series with regard to the variable $x$. Therefore, it is sufficient to demonstrate that the coefficients of the Fourier series of the functions on the left hand sides correspond to coefficients on the right hand sides. This demonstration is easy because the right hand sides are trigonometric functions. Then, by combining equations (41),(42) and (43) we obtain

$$
\begin{aligned}
\eta(x)=\frac{1}{4 \lambda_{e}^{3} E I} & {\left[\frac{\mathrm{e}^{\mathrm{i} \omega \frac{x}{v}} \sin \lambda_{e}(L-x)+\mathrm{e}^{-\mathrm{i} \omega \frac{L-x}{v}} \sin \lambda_{e} x}{\cos L \lambda_{e}-\cos \frac{\omega L}{v}}-\right.} \\
& \left.\frac{\mathrm{e}^{\mathrm{i} \omega \frac{x}{v}} \sinh \lambda_{e}(L-x)+\mathrm{e}^{-\mathrm{i} \omega \frac{L-x}{v}} \sinh \lambda_{e} x}{\cosh L \lambda_{e}-\cos \frac{\omega L}{v}}\right]
\end{aligned}
$$

Particularly, when $L=m l$ and $x=p l$, we have $\eta_{p}=\eta(p l)$.

\section{Appendix B. Calculation of expression $\chi_{p}$}

By setting $n=r m+p$ with $0 \leq p \leq m-1$ and $r \in \mathbb{Z}$, we can develop equation (14) as follows:

$$
\begin{aligned}
\eta_{p} & =\frac{1}{L E I} \sum_{n \in \mathbb{Z}} \frac{e^{i 2 \pi n \frac{p}{m}}}{\left(\frac{\omega}{v}+\frac{2 \pi n}{L}\right)^{4}-\lambda_{e}^{4}} \\
& =\sum_{q=0}^{m-1} \frac{1}{L E I} \sum_{r=-\infty}^{\infty} \frac{e^{i 2 \pi(m r+q) \frac{p}{m}}}{\left(\frac{\omega}{v}+\frac{2 \pi(m r+q)}{L}\right)^{4}-\lambda_{e}^{4}} \\
& =\sum_{q=0}^{m-1} \frac{e^{i 2 \pi \frac{p q}{m}}}{m l E I} \sum_{r=-\infty}^{\infty} \frac{1}{\left(\frac{\omega}{v}+\frac{2 \pi(m r+q)}{L}\right)^{4}-\lambda_{e}^{4}}
\end{aligned}
$$

In addition, we have

$$
\begin{aligned}
\chi_{q} & =\frac{1}{l E I} \sum_{r=-\infty}^{\infty} \frac{1}{\left(\frac{\omega}{v}+\frac{2 \pi(m r+q)}{L}\right)^{4}-\lambda_{e}^{4}} \\
& =\frac{1}{2 \lambda_{e}^{3} E I} \sum_{r=-\infty}^{\infty} \frac{l \lambda_{e}}{\left(l \frac{\omega}{v}+2 \pi\left(r+\frac{q}{m}\right)\right)^{2}-\left(l \lambda_{e}\right)^{2}}-\frac{l \lambda_{e}}{\left(l \frac{\omega}{v}+2 \pi\left(r+\frac{q}{m}\right)\right)^{2}+\left(l \lambda_{e}\right)^{2}} \\
& =\frac{1}{4 \lambda_{e}^{3} E I}\left(\frac{\sin l \lambda_{e}}{\cos l \lambda_{e}-\cos \left(\frac{\omega l}{v}+\frac{2 \pi q}{m}\right)}-\frac{\sinh l \lambda_{e}}{\cosh l \lambda_{e}-\cos \left(\frac{\omega l}{v}+\frac{2 \pi q}{m}\right)}\right)
\end{aligned}
$$

Thus, we have

$$
\eta_{p}=\frac{1}{m} \sum_{q=0}^{m-1} \mathrm{e}^{\mathrm{i} 2 \pi \frac{p q}{m}} \chi_{q}
$$

where $\chi_{p}$ is defined by equation (45). 


\section{References}

[1] D.J. Mead, "Free wave propagation in periodically supported, infinite beams", Journal of Sound and Vibration, 11(2): 181-197, 1970.

[2] P.M. Belotserkovskiy, "On the oscillation of infinite periodic beams subjected to a moving concentrated force", Journal of Sound and Vibration, 193(3): 705-712, 1996.

[3] A. Nordborg, "Vertical Rail Vibrations: Pointforce Excitation", Acustica, 84: 280-288, 1998.

[4] "Vertical Rail Vibrations: Parametric Excitation", Acustica, 84: 289-300, 1998.

[5] A.V. Metrikine, K. Popp, "Vibration of a periodically supported beam on an elastic half-space", European Journal of Mechanics A/Solid, 18: 679-701, 1999.

[6] A.V. Vostroukhov, A. Metrikine, "Periodically supported beam on a visco-elastic layer as a model for dynamic analysis of a high-speed railway track", International Journal of Solids and Structures, 40: 5723-5752, 2003.

[7] T. Hoang, D. Duhamel, G. Foret, H. Yin, P. Joyez, R. Caby, "Dynamical response of railway tracks in tunnel", $11^{\text {th }}$ World Congress on Computational Mechanics (WCCM XI-ECCM V-ECFD VI), July 20-25, 2014, Barcelona, Spain.

[8] R. Bracewell, The Fourier transform and its applications, McGraw-Hill Higher Education, 2000, ISBN 9780073039381 , LCCN 99021139. 